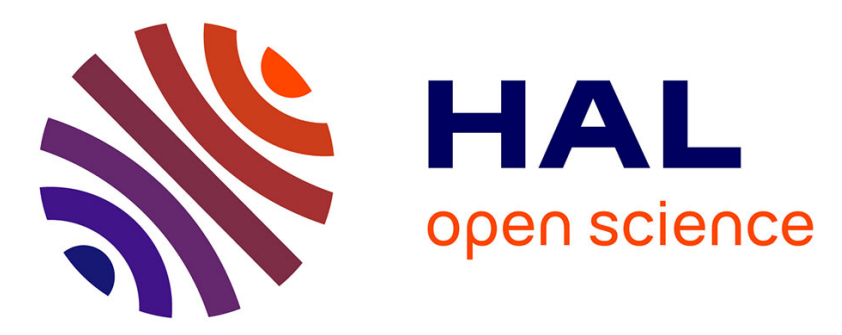

\title{
Retour sur une expérience a priori inhabituelle : former des futurs enseignants de français langue étrangère à l'exploitation de Moodle avec Moodle
}

\author{
Jean-Luc Pierre Bergey
}

\section{- To cite this version:}

Jean-Luc Pierre Bergey. Retour sur une expérience a priori inhabituelle : former des futurs enseignants de français langue étrangère à l'exploitation de Moodle avec Moodle. Recherche et pratiques pédagogiques en langues de spécialité - Cahiers de l'APLIUT, 2015, XXXIV (N 1), pp.181 - 195. 10.4000/apliut.5070 . hal-01727121

\section{HAL Id: hal-01727121 \\ https://hal.science/hal-01727121}

Submitted on 9 May 2021

HAL is a multi-disciplinary open access archive for the deposit and dissemination of scientific research documents, whether they are published or not. The documents may come from teaching and research institutions in France or abroad, or from public or private research centers.
L'archive ouverte pluridisciplinaire HAL, est destinée au dépôt et à la diffusion de documents scientifiques de niveau recherche, publiés ou non, émanant des établissements d'enseignement et de recherche français ou étrangers, des laboratoires publics ou privés. 
Cahiers de l'Apliut

Vol. XXXIV No 1 | 2015

La formation en langues/LANSAD dans les centres de langues : état des lieux et perspectives

\section{Retour sur une expérience a priori inhabituelle : former des futurs enseignants de français langue étrangère à l'exploitation de Moodle avec Moodle} Revisiting a seemingly unusual experiment: training future teachers of French as a Foreign Language to exploit Moodle thanks to Moodle

\section{OpenEdition} Journals

Édition électronique

URL : http://journals.openedition.org/apliut/5070

DOI : 10.4000/apliut.5070

ISSN : 2119-5242

Éditeur

APLIUT

Édition imprimée

Date de publication : 15 janvier 2015

Pagination : 181-195

ISBN : 978-2-8218-5355-3

ISSN : 2257-5405

Ce document vous est offert par Université Bordeaux Montaigne 

Retour sur une expérience a priori
inhabituelle : former des futurs
enseignants de français langue étrangère
à l'exploitation de Moodle avec Moodle

Mots-clés

plate-forme d'apprentissage, formation, TICE, FLE, ingénierie pédagogique, approche cognitive, pratique réflexive, apprentissage composite

\section{Résumé}

Depuis 2007, dans le cadre du Master DiFLES (didactique du français langue étrangère et seconde) à BordeauxMontaigne, nous proposons une offre de cours en ligne (à partir de la plateforme d'apprentissage Moodle) en complément des cours présentiels. Les enjeux s'y sont avérés au moins doubles :

- apprendre le cœur du métier d'enseignant de FLE et le rôle d'apprenant-utilisateur de Moodle impliqué dans sa propre formation ;

- proposer, en $2^{\mathrm{e}}$ année, une approche pratique du rôle d'administrateur-concepteur de projet pédagogique avec Moodle.

Or, un troisième enjeu est apparu: (re)penser l'espace Moodle au-delà d'un cadre traditionnel d'accompagnement de cours présentiels. Nos observations d'ordre technico-pédagogique concernent l'expérience d'un groupe de collaboration exploitant Moodle comme instrument de formation réflexif et support d'expérimentation pour une ingénierie de formation.

\section{Revisiting a seemingly unusual experiment: training future teachers of French as a Foreign Language to exploit Moodle thanks to Moodle}

\section{Keywords}

LMS, ICT, FFL, pedagogical engineering, cognitive approach, reflective learning, blended learning

\section{Abstract}

Since 2007, in the DiFLES Master's degree program (teaching of French as a Foreign and Second Language) we have been offering access to online courses (thanks to the LMS Moodle) in addition to classroom courses. The issue has proven to be twofold:

- learning the core tenets of FFL teaching, and learning about the role of a learner-user of Moodle involved in his/her own training;

- in the 2nd year, providing a practical approach to the role of administratordesigner of pedagogical projects with Moodle.

Now a third concern has appeared: (re)defining the use of Moodle which, so far, has been a support system for classroombased courses. We call for technical and pedagogic observations, as we experimented it with a working group and using it both as a training device and a tool for reflection since Moodle was also a ground for training in the field of pedagogic engineering. 


\section{Retour sur une expérience a priori inhabituelle : former des futurs enseignants de français langue étrangère à l'exploitation de Moodle avec Moodle}

\section{Introduction}

\section{Appréhender une pléthore d'outils technico-pédagogiques}

Un futur enseignant de FLE ne peut ignorer la pléthore d'outils technico-pédagogiques désormais présente dans les institutions d'enseignement des langues.

Les environnements numériques de travail $\left(\mathbf{E N T}^{1}\right)$, sites institutionnels, intègrent des fonctionnalités diverses et souvent une dimension d'enseignement-apprentissage (désormais E/A). Les CMS (Content Management System) populaires mettent en œuvre des environnements diversifiés: sites institutionnels, ENT, et blogs par exemple. Les LMS (Learning Management System) intègrent la dimension learning comme spécialisation et prévoient une batterie de moyens favorisant 1 ' $E / \mathrm{A}^{2}$. Néanmoins, comme le signale Cédric Sarré (2011: 16), il existe des choix qui vont «permettre de qualifier l'approche adoptée », notamment quant au «statut accordé aux connaissances» et «le rôle des apprenants dans la construction de leurs connaissances.». Ainsi les approches pourront-elles être «transmissive», « individualiste » ou « collaborative » (Charlier et al. $2006: 482$ ).

\section{Une évolution constante des pratiques et dispositifs numériques}

Les réseaux sociaux ont vu de nombreuses expérimentations justifier leur usage dans la classe de langue (Facebook ou Twitter). Les pratiques de réseautage sont liées comme le signale Katerina Zourou (2012) à trois caractéristiques majeures de l'évolution du web (participation de l'utilisateur, ouverture et effets de réseautage). La deuxième génération de dispositifs du web, dite « $2.0 »$, illustre bien cette évolution par l'apparition de communautés de pratique d'apprentissage en ligne: par exemple, les sites «Busuu » ou «Live Mocha », opportunément mis en valeur dans les parcours d'apprentissage d'apprenants de langue seconde (Ollivier \& Puren 2011). Dans ce courant, la ludification d'activités d'apprentissage s'est également développée à travers les serious games.

\footnotetext{
${ }^{1}$ Le sigle ENT ne désigne pas un type de logiciel comme LMS et CMS. Mais l'ENT peut avoir été conçu (entre autres) avec l'un de ces derniers.

${ }^{2}$ Moodle, Claroline, Dokeos sont des LMS populaires, Moodle revendique une orientation pédagogique socioconstructiviste.
} 
En vogue actuellement, les MOOC (Massive Open Online Courses) proposent une pédagogie en ligne, initialement conçue comme massive et nécessairement transmissive ${ }^{3}$. En reprenant les propos de Marcel Lebrun, Christine Vaufrey (2011) souligne que dans ce modèle initial « xMOOC» :

\begin{abstract}
L'enseignant y tient clairement le premier rôle, dans des interventions enregistrées à l'avance qui excluent toute communication synchrone avec les apprenants. Comment pourrait-il d'ailleurs y avoir une communication avec une équipe composée de 6 ou 8 pédagogues (l'enseignant et ses « teaching assistants») face à des dizaines ou centaines de milliers de participants?
\end{abstract}

En ajoutant un «c», par référence au concept de Connective Knowledge (Downes 2012), les «cMOOC» proposent une structuration du dispositif au profit d'une approche collaborative ${ }^{4}$. Un arsenal déjà riche de possibilités, auquel on peut ajouter les TNI (tableaux numériques interactifs) et la baladodiffusion. Enfin, l'évolution des terminaux de type smartphone a entraîné également d'autres évolutions des pratiques. Car « la forte expansion de l'Internet et du haut débit a permis de s'émanciper de ces 'Powerpoint améliorés' », rappelle Jean-Christophe Coquilhat (2008 : 95). Un courant de Mobile Assisted Language Learning (MALL) s'empare du développement d'applications pour smartphones. Malgré la taille des écrans (quantité d'informations limitée), nous y voyons, comme Glenn Stockwell, un intérêt indéniable :

L'apprentissage à partir de terminaux mobiles permet non seulement d'augmenter
potentiellement le temps que consacre chaque apprenant aux activités linguistiques, mais
aussi de réduire l'écart psychologique qui peut être associé à des situations plus formelles
d'apprentissage de langue $^{5}$. (2012:201, notre traduction)

Ces techniques se rationnalisent et s'institutionnalisent parallèlement aux usages privés (MySpace, Second Life ; Twitter, Facebook) ou identifiés comme des réseaux professionnels (Ning, LinkedIn, Viadeo, Google ${ }^{6}$ ). Or, la nature des échanges qui s'y pratiquent ne relève, le plus souvent, que du partage d'informations, et leur aspect plus ou moins formel en réduit les possibilités pour des dispositifs d'E/A rationnalisés et autonomes.

\footnotetext{
${ }^{3}$ Le modèle initial (voir CourseEra, EdX, Udacity) a permis à des universités prestigieuses de diffuser une offre de cours alternative : Stanford, Berkeley, Harvard, MIT ont popularisé ce modèle.

${ }^{4}$ Marcel Lebrun et Christophe Batier ont lancé, en novembre 2014, un «x-c-Mooc » à partir du LMS claroline connect.

5 «Mobile learning has the potential to not only increase the amount of time that individual learners spend engaged in language activities, but also to reduce the psychological distance that maybe associated with more formal language learning situations ». (Stockwell $2012: 201$ ).

${ }^{6}$ Google + a la particularité d'être « grand public » comme Facebook, mais structurable en « cercles » de sous-réseaux séparés (professionnels, amicaux, familiaux ou informels).
} 


\section{Quels enjeux pour la formation?}

\subsection{De l'utilisateur au didacticien}

Administrant un dispositif Moodle depuis 2008 (Master Pro DiFLES ${ }^{7}$ ), nous avons animé un cours intitulé " Dispositif de formation ouverte et à distance» comme un lieu d'expérimentation du potentiel des plateformes LMS. Notre objectif était de lier deux rôles complémentaires du métier d'enseignant de FLE :

- l'utilisateur des outils des TICE (technologies de l'information et de la communication pour l'enseignement), qui s'empare des possibilités offertes par les institutions d'accueil (exploitation rationnelle des ressources et outils offerts par l'institution d'E/A);

- puis, le didacticien impliqué «en amont», intégrant une posture réflexive dès l'élaboration de ressources, leur scénarisation et la planification d'un curriculum.

Comme il existe des rôles intermédiaires qui ne sont absolument pas triviaux (coordination d'équipe, gestion des ressources d'E/A, organisation des actions, adéquation avec un curriculum, etc.), notre schéma, figure 1, est un continuum : la flèche ascendante illustre un niveau d'expertise ; l' « expert » dans l'exploitation des ressources et outils de l'institution peut non seulement gérer-adapter des ressources d'E/A, mais en progressant vers des compétences de didacticien, également modifieradapter des scenarii et/ou curricula existants.

Figure 1. De l'utilisateur au didacticien : une « traversée du miroir ${ }^{8}$

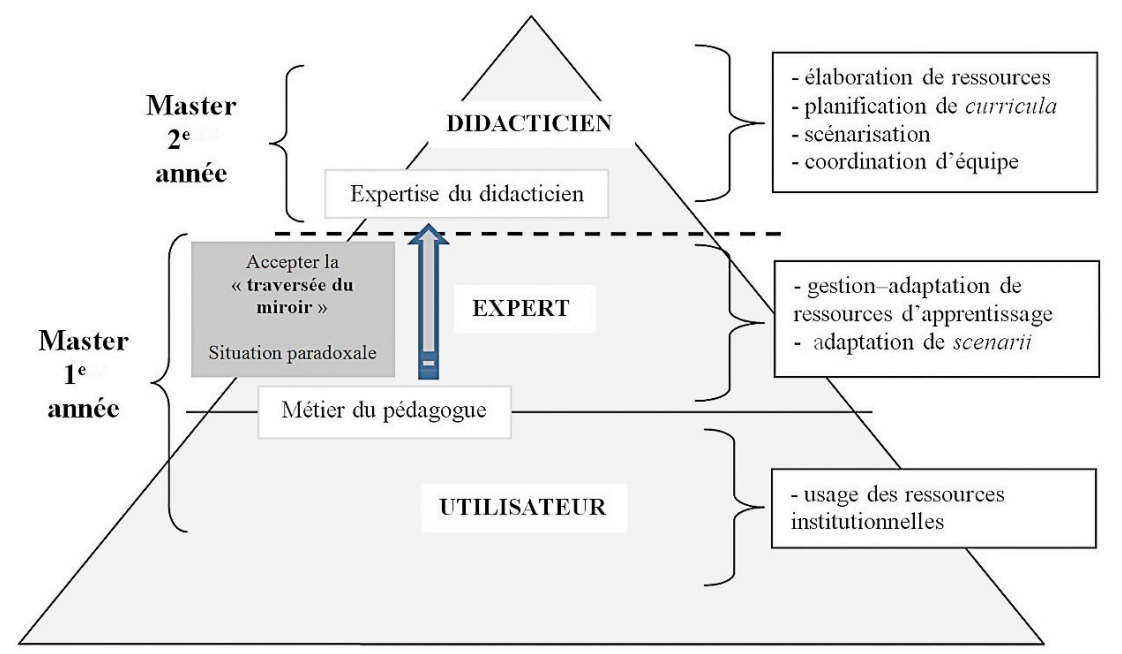

\footnotetext{
${ }^{7}$ Le Master professionnalisé «DiFLES » comprend deux parcours distincts en deuxième année, notre communication concerne le groupe ILF «Ingénierie linguistique et de formation »: <http://www.ubordeaux-montaigne.fr/fr/formations/offre_de_formation/master-XB/arts-lettres-langues-ALL/masterprofessionnel-didactique-fles-program-mnl-21.html>

${ }^{8}$ Les figures 1 à 5 sont des illustrations originales de l'auteur.
} 
Notre Master est organisé ainsi : la pédagogie en $1^{\mathrm{e}}$ année, et une approche plus didactique en $2^{\mathrm{e}}$ année car fondée sur un questionnement disciplinaire en contexte numérique. La deuxième année est une étape clef, avec un deuxième semestre exclusivement dédié à un stage professionnel de 470 heures.

Mettre en contact didactique et pédagogie permet une adaptation au marché du travail (contexte numérique) et une réponse à des contextes d'enseignement variés ${ }^{9}$. Nous insistons sur les points suivants :

- dépasser une formation « traditionnelle» des enseignants de FLE (détenteur d'un savoir disciplinaire fondé sur du « transmissif ») ;

- intégrer des compétences de "personne ressource TICE », pour un savoir " procédural » permettant d'intervenir à des niveaux divers des formations ;

- articuler ces deux niveaux de compétence pour enrichir l'offre de formation «pro » du Master ;

- promouvoir la conception technico-pédagogique (intégration de plugins, scénarisation numérique de curriculum existant, etc.).

L'implantation croissante de Moodle et l'absence de formations adaptées aux équipes pédagogiques ont conduit certains de nos étudiants à assumer le rôle d'ingénieur pédagogique au cours de leur stage et après ${ }^{10}$.

\subsection{Accepter une situation inhabituelle, pour un nouveau rôle}

Lors du passage entre la $1^{\mathrm{e}}$ et la $2^{\mathrm{e}}$ année, la «traversée du miroir » (cf. figure 1), il s'est agi d'adopter des comportements nouveaux en acceptant une alternance de rôles dans des phases comme «enseigné », sous la tutelle classique de l'enseignant, et des phases d'exercice d'une expertise d' « enseignants » encore en devenir (cf. figure 2).

Figure 2. Alternance de rôles

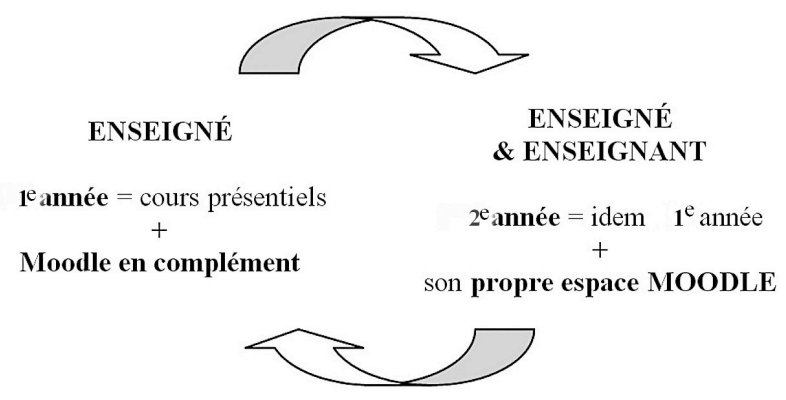

\footnotetext{
${ }^{9}$ En terme d'exigences, d'organisation et de publics d'apprenants, les institutions d'accueil de nos étudiants ont permis des stages de fin de Master stimulants: école d'ingénieurs (ENSEIRB-MATMECA) en anglais professionnel (ESP), IEP de Bordeaux ou écoles privées (École Supérieure de Langue Franco-Japonaise à Paris). Mais aussi à l'étranger : GSÜ-Galatasaray à Istanbul et réseaux d'alliances françaises.

${ }^{10}$ Une de nos diplômées en 2012 a été recrutée comme ingénieur pédagogique au sein de 1'Université d'Auvergne.
} 
Il s'agissait pour nos étudiants d'une situation «paradoxale », en raison de difficultés prégnantes à assumer une posture d'enseignant, due selon nous à une culture transmissive du savoir et des enseignements. Nous souhaitions une attitude plus réflexive focalisant sur «le rôle des processus de reconstruction, comprenant la métacognition, une articulation consciente au fil du temps, et une réflexion sur l'apprentissage $"{ }^{11}$ (Hubbard 2008: 183; notre traduction). Comme le suggère Nicolas Guichon, l'attitude rétrospective « permet aux apprentis de s'approprier peu à peu leur métier, de construire une identité professionnelle » (Guichon $2011: \S 12$ ); en conséquence, la pratique professionnelle

[...] a besoin d'être apprivoisée tout d'abord en plaçant l'apprenti dans une situation réelle de travail puis en ménageant des moments où cette situation sera reprise, arpentée et examinée par l'apprenti de manière à le conduire à ajuster son activité pour qu'elle gagne en qualité dans une situation semblable à venir.

Il s'agissait, d'acquérir une «technique de soi » (Foucault 1988), pour «permettre de se construire une représentation de soi au travail et de se forger un discours en même temps qu'une identité » (Guichon 2011: §3). Comme le souligne encore Nicolas Guichon :

[...] au-delà de l'analyse critique que permet ce type de dispositif, la rétrospection installe l'apprenti dans une éthique professionnelle, une sorte d'inconfort initial qui incite l'apprenti, futur professionnel, à résister à l'évidence de la pratique et à l'autosatisfaction. (idem :\$64.)

\section{Quels résultats?}

\subsection{Un cadre spécifique au parcours en Master DiFLES}

D'une part, il s'agissait de s'écarter d'une exploitation de « recettes » pédagogiques (Stanford 2009), que nous jugions contre-productive et peu créative. D'autre part, la réalisation autonome de cours s'éloignait d'un comportement appris : « une déférence au maître, l'obéissance aux règles et aux consignes, et par l'imitation / la restitution des savoirs transmis par l'enseignant » (Vaufrey 2013). Cette attitude renvoie trop directement aux méthodes transmissives mentionnées précédemment (Charlier et al. 2006).

Les technologies sont certes porteuses de potentiels pour le développement pédagogique mais, afin d'en retirer les valeurs pédagogiques espérées, elles nécessitent d'être encadrées par des dispositifs pédagogiques basés sur des méthodes plus incitatives et interactives [...]. (Lebrun 2011)

À l'instar d'une célèbre marque suédoise de meubles en kit, notre objectif était de dépasser l'étape d'assemblage à partir d'une notice-recette, pour certes, élaborer un «objet-cours », mais en ayant pensé et produit au préalable sa (ses) propre(s) notice(s) d'assemblage de cet « objet-cours » (cf. figure 3).

\footnotetext{
${ }^{11}$ «[...] the role of reconstructive processes, including metacognition, conscious articulation over time, and reflection on apprenticeship ». (Hubbard $2008: 183$ )
} 
Figure 3. Constat d'un parcours en Master DiFLES

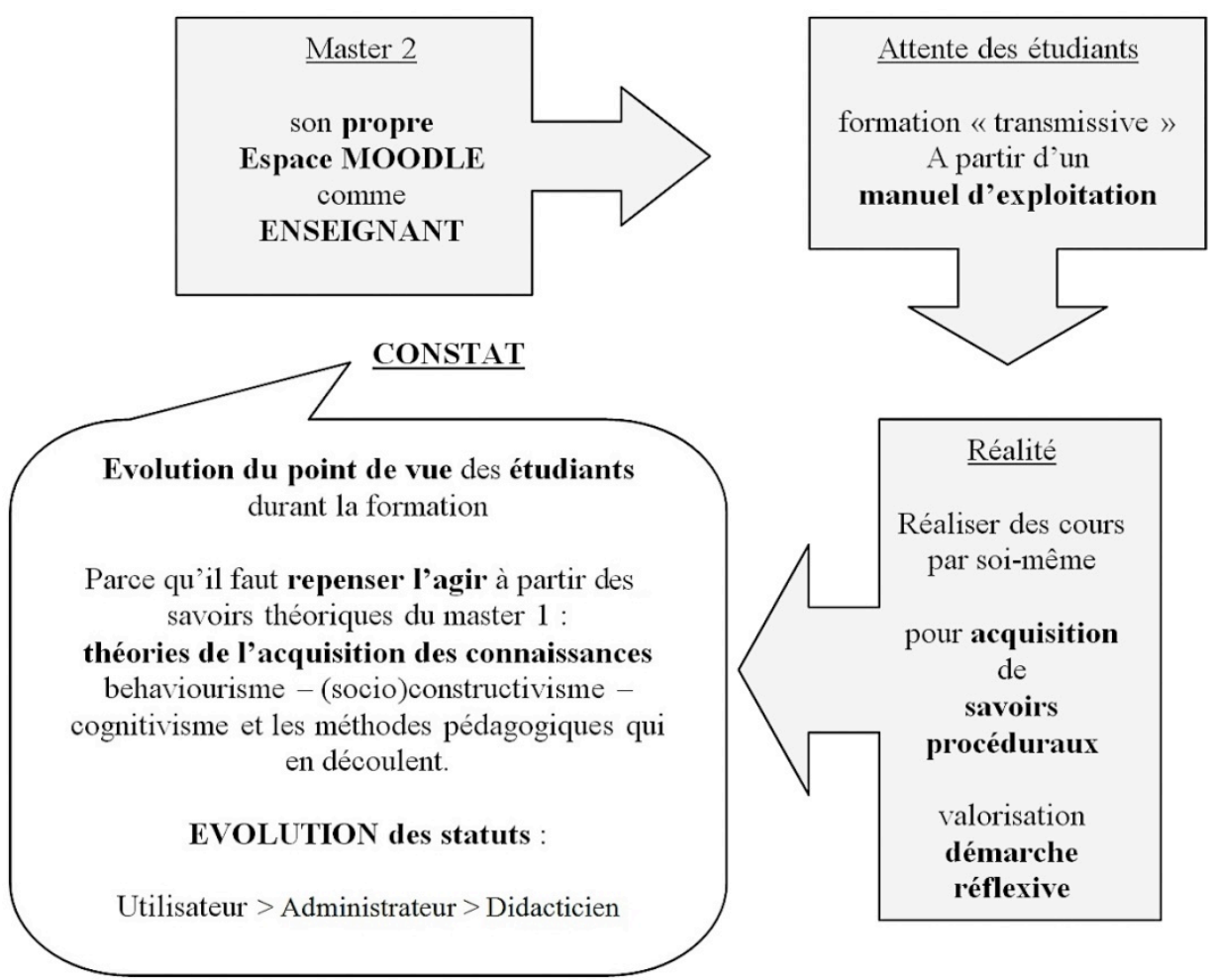

Réaliser des cours par soi-même a permis un lien avec les contenus théoriques de première année du Master (behaviourisme - (socio)constructivisme - cognitivisme). L'évolution du point de vue des étudiants est passée par l'acquisition de savoirs procéduraux et une valorisation de la démarche réflexive (pratiques - analyse (ré)ajustements); d'autres gains qualitatifs ont été observés :

- une réflexion accrue sur l'outil Moodle et son articulation avec les TICE;

- l'émergence d'une expertise technico-pédagogique ;

- un passage progressif d'une logique de transmission d'informations à une logique d'interaction et de scénarisation.

En effet, nos étudiants ont constitué un réseau d'échanges de procédures de résolution de problèmes, en fonction des contextes de formation ${ }^{12}$ sur lesquels ils travaillaient. Ils sont passés progressivement des espaces de partages d'information, aux espaces collaboratifs d'échanges de savoirs procéduraux.

\footnotetext{
${ }^{12}$ Les étudiants sont évalués sur une «étude de cas », un cours complet avec Moodle en fonction de contextes réels de stages.
} 


\subsection{Des espaces pour collaborer - coopérer}

Utiliser un «forum» dans Moodle génère des interactions limitées, en raison d'échanges majoritairement à un niveau «déclaratif » ${ }^{13}$. Tandis que le «wiki»d'emblée orienté vers la co-création de contenus - inviterait plutôt à des interactions de type «procédural» :

Cet espace de co-construction d'un objet, élaboré au sein d'une collectivité, fait aussi du wiki un processus d'évaluation croisée : chaque contribution comporte explicitement ou implicitement une évaluation des contributions déjà produites; chaque individu se constitue ainsi comme membre à part entière d'une communauté. (Bergey et al. 2012: $54)$.

À propos des outils issus du web 2.0, Gràinne Conole et Panagiota Alevizou soulignent également une synergie productive entre communication et co-création :

L'importance de la collaboration est un ingrédient commun dans la plupart des perspectives d'apprentissage, au point de la considérer généralement comme un élément important pour le développement de la compréhension dans le dialogue et la coconstruction. ${ }^{14}$ (2010: 16 ; notre traduction).

Pour ces derniers, le wiki constitue un outil particulièrement adapté à la collaboration :

[...] le potentiel des wikis pour faciliter l'apprentissage coopératif dans un environnement constructiviste et pour promouvoir des communautés de pratique a été avancé. Les wikis sont considérés comme des outils idéaux pour l'écriture collaborative et pour l'étayage de projets de groupe. ${ }^{15}$ (idem : 30 ; notre traduction).

En distinguant l'apprentissage collaboratif de l'apprentissage coopératif, Alain Baudrit (2007: 126) met en évidence des coordinations sociales différentes : « les échanges spontanés, non structurés au départ, propres de l'apprentissage collaboratif font penser à des coordinations sociales de type "partage" », tandis que « les relations organisées, voire préconçues, spécifiques de l'apprentissage coopératif portent à croire que nous sommes en présence de coordinations sociales de type "assemblage" ». Selon lui, « ces deux méthodes ne devraient pas servir les mêmes objectifs, viser les mêmes types d'apprentissage » (ibidem).

Outre les outils mis en œuvre pour offrir des espaces de partage, des phasages et des articulations de tâches seraient à prévoir pour des objectifs différents, mais complémentaires : des moments de collaboration «partage » où chacun apporte ses savoirs et ses connaissances pour avancer dans l'organisation et la progression d'une

\footnotetext{
${ }^{13}$ Le «forum » Moodle autorise les pièces jointes, mais l'aspect antéchronologique et éclaté de son interface limite le partage spontané de procédures de résolution de problème.

14 " The importance of collaboration is a common ingredient in many of the learning perspectives, as it is generally considered to be an important means of developing understanding through shared dialogue and co-construction ». (Conole \& Alevizou $2010: 16$ )

15 «[...] the potential of wikis for facilitating cooperative learning in a constructivist environment and for fostering communities of practice has been argued. Wikis are considered to be ideal tools for collaborative writing and for scaffolding group projects ». (Conole \& Alevizou 2010 : 30)
} 
activité, et des «assemblages »- lorsque chacun maîtrise une partie du tout, une part précise du travail global à réaliser - qui requièrent des savoir-faire opératoires. Roy Lewis note également que :

[...] la création de représentations abstraites (règles ou visualisation) est une clé pour la résolution collective de problèmes ; par exemple, les étudiants qui dessinent des schémas pour représenter le problème qu'ils doivent résoudre réussissent mieux que ceux qui ne le font pas. L'action de dessiner un schéma grossier permet de construire une représentation commune du problème ; les apprenants créent donc ainsi un mécanisme de 'construction d'une représentation partagée'. (1998: 17)

\section{Observation « technico-pédagogique » d'un déséquilibre}

\subsection{Les trois pôles de Moodle}

Comme la figure 4 ci-dessous le montre, l'exploitation de Moodle révèle un déséquilibre manifeste: un pôle communication surreprésenté et fondé sur des interactions relativement faibles (au niveau du « déclaratif») qui est le résultat de l'exploitation des activités « chat » et « forum », et de contenus de cours numérisés (des « objets », contenus transmissifs). Un pôle dédié à l'apprentissage s'appuie sur des ressources documentaires transmissives à partir de « leçon » et « test » associées à des exerciseurs à visée évaluative (HotPotatoes, Netquiz). Or, ces derniers sont utilisés dans un cadre behaviouriste (exercices de type «stimulus-réponse») qui, bien qu'étant nécessaires, renvoient à des opérations de « bas-niveau ${ }^{16}$.

Des opérations de « haut-niveau » supposeraient des activités cognitives comprenant des phasages de planification-actions-ajustements, plus évidentes à susciter lors d'activités coopératives-collaboratives ou, par exemple, des activités discursives situées. Prouver l'efficacité pédagogique des LMS se heurte - comme le remarque Lebrun - à «des résultats peu probants et peu décisifs ». Se référant à Benjamin Bloom et al. (1956), Lebrun ajoute «que les effets recherchés (en termes de compétences et de savoir-être) restent hors de portée des évaluations certificatives encore largement basées sur des compétences de bas niveau comme la restitution ou l'application » $(2011: 2)$.

\footnotetext{
${ }^{16}$ L'expression «bas niveau » due à Lindsay \& Norman (1980) désigne un traitement perceptif à partir des données (par exemple, des activités d'automatisation de micro-procédures linguistiques), à la différence d'un traitement de « haut-niveau » à partir des concepts.
} 
Figure 4. Orientation des 3 pôles

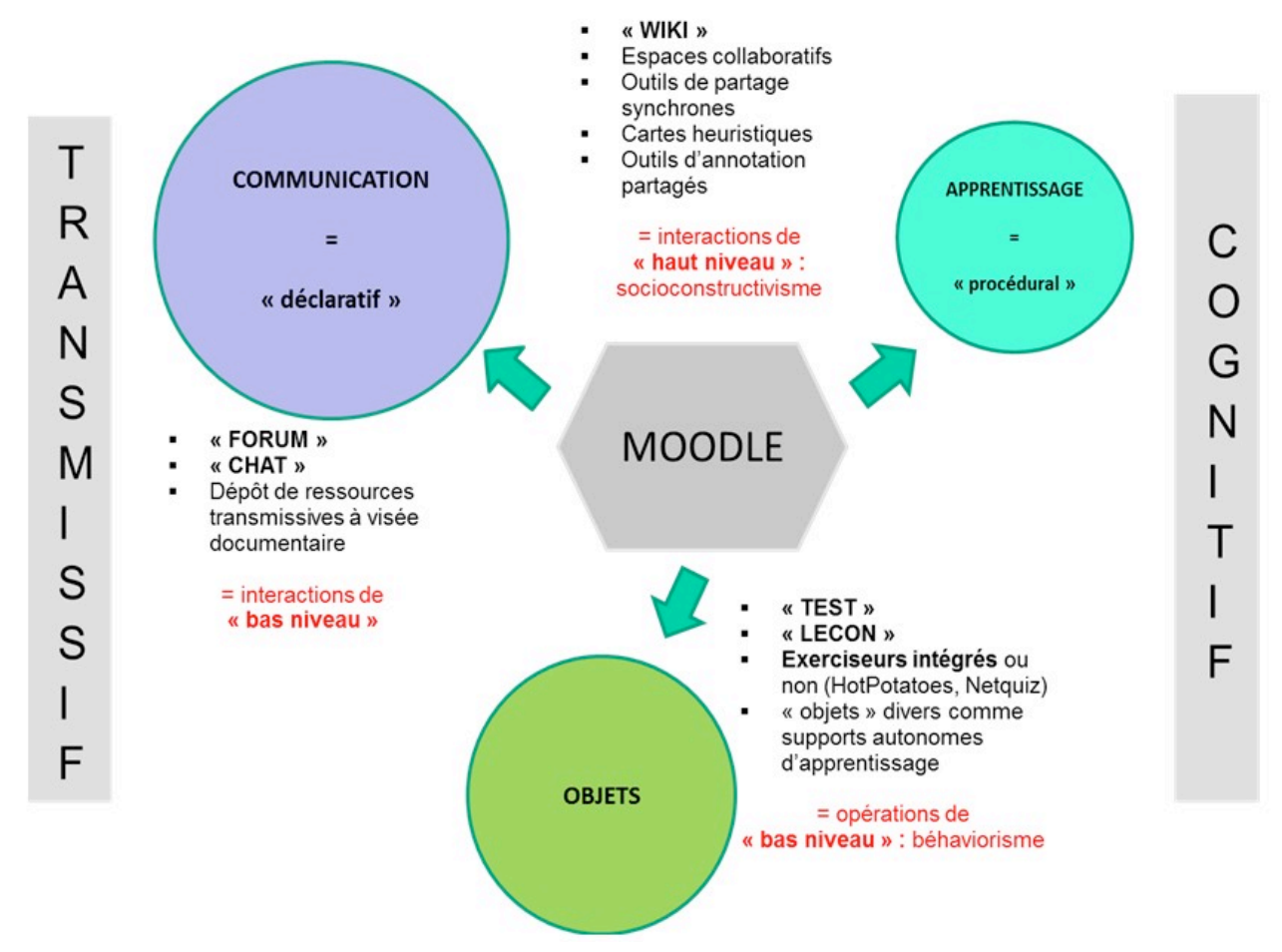

\subsection{Un rééquilibrage nécessaire}

Le pôle «apprentissage », sous-représenté, traduit une difficulté à investir le "côté cognitif ». Ce déséquilibre conduit Lebrun à parler de TMS $^{17}$ (Teaching Management System), en raison d'une structuration exposant surtout le savoir-faire «transmissif»" indéniable de l'enseignant. Un effort de scénarisation devrait rééquilibrer l'apprentissage vers un travail cognitif, au profit du pôle « apprentissage ».

Cette difficulté est associée, selon Pascal Béguin et Pierre Rabardel, à l'appréhension de «l'instrument médiateur » comme une « entité mixte» (2000:42). Un instrument, constitué de deux types de structures : «des structures psychologiques organisatrices de l'activité » et "des structures de type artefacts»; " ces deux structures sont solidairement constitutives de la médiation à l'objet de l'activité. » (ibidem). Aussi, les auteurs prônent une approche anthropocentrée des objets techniques, au service des activités d'apprentissage.

17 Blog de Lebrun : <http://lebrunremy.be/WordPress/?p=629\#comments> (consulté le 15 novembre 2014).

Recherche et pratiques pédagogiques en langues de spécialité - Cahiers de l'APLIUT Volume XXXIV No 1 , janvier 2015 - ISSN : 2257-5405 


\section{Une scénarisation composite}

\subsection{Le concept d'une scénarisation composite}

Figure 5. Rééquilibrage et composition des 3 pôles
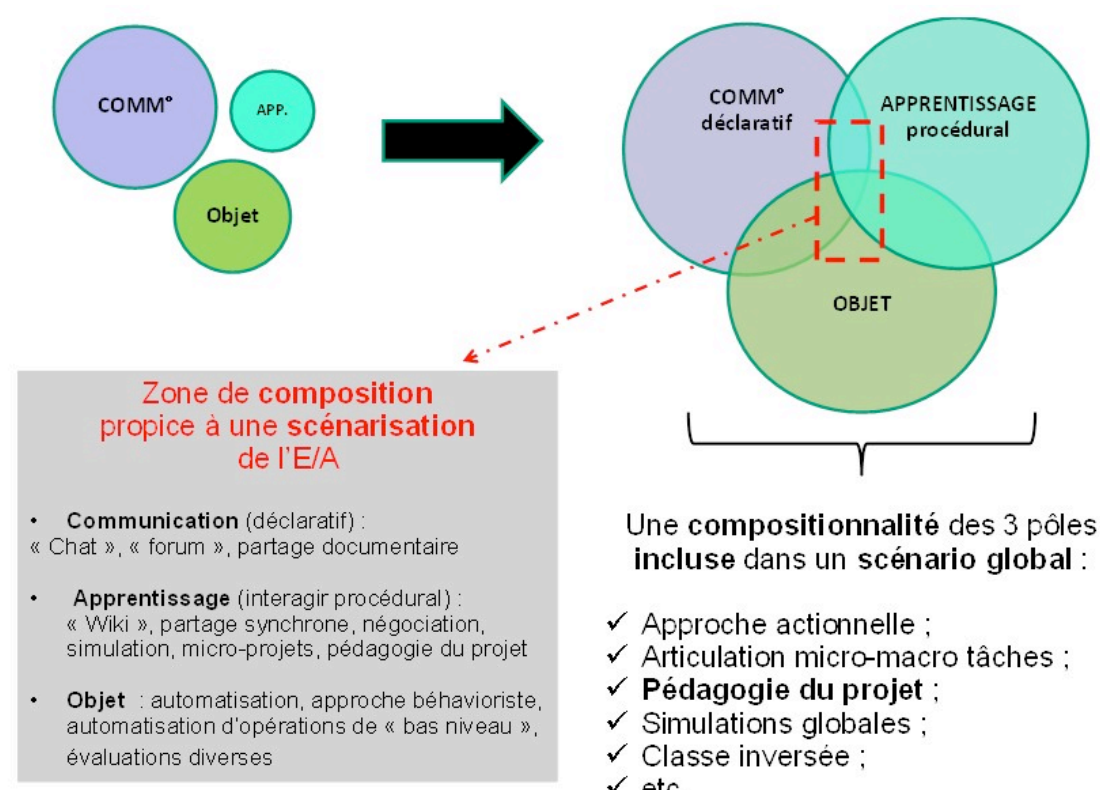

Une compositionnalité des 3 pôles incluse dans un scénario global :

$\checkmark$ Approche actionnelle ;

$\checkmark$ Articulation micro-macro tâches:

$\checkmark$ Pédagogie du projet;

$\checkmark$ Simulations globales:

$\checkmark$ Classe inversee ;

$\checkmark$ etc.

L'approche actionnelle et la pédagogie du projet s'avèrent adaptées, grâce à un ancrage social des tâches « à savoir la prise en compte de l'agir social aussi bien en société que dans l'espace même de la classe », qui permet de considérer «les activités d'apprenants dans l'espace et le temps même de leur apprentissage, comme des acteurs sociaux à part entière »(Puren 2009: 120-121). Comme le dit Christian Ollivier :

C'est dans la non évocation des tâches proposées dans la vie réelle que réside une des limites les plus sensibles de la perspective actionnelle qui pense essentiellement en termes de tâches 'proches de la vie réelle' et de 'tâches didactiques' et éclipse ainsi l'un des facteurs les plus déterminants de toute action: la contrainte relationnelle dans laquelle l'action s'opère. (2009: 266).

\subsection{Une approche pragmatique?}

Le tableau croisé $^{18}$ de Joyce Seitzinger (2010), traduit par Nicolas Martignoni ${ }^{19}$

\footnotetext{
${ }^{18}$ Tableau originel de Joyce Seitzinger :

$<$ http://www.cats-pyjamas.net/wp-content/uploads/2010/05/MoodleToolGuideforTeachers_May2010_JS.pdf $>$ (consulté le 15 novembre 2014).

${ }^{19}$ Traduction du tableau de Joyce Seitzinger par Nicolas Martignoni (consulté le 15 novembre 2014) : $<$ https://moodle.org/pluginfile.php/1348613/mod_resource/content/2/MoodleToolGuide_fr.pdf $>$.
} 
(2013), (« ce que vous voulez faire» versus « ce que vous voulez utiliser»), permet d'aborder les outils de Moodle en contournant les écueils d'une prise en main triviale.

Dans les deux extraits du tableau ci-dessous (figures 6 et 7), les quatre premiers items «ce que vous voulez utiliser» en haut suggèrent une progression, du «transfert d'information » à la " co-création de contenus ", soit d'une pédagogie transmissive, à une collaboration-coopération assumée. Un cinquième item (en haut à droite) replace chaque activité par rapport aux six niveaux de la taxonomie ${ }^{20}$ de Bloom et al. (1956). Une indication (première forme fond blanc en haut à gauche) de «facilité d'emploi » est proposée.

Figure 6. Tableau croisé Seitzinger-Martignoni (1)

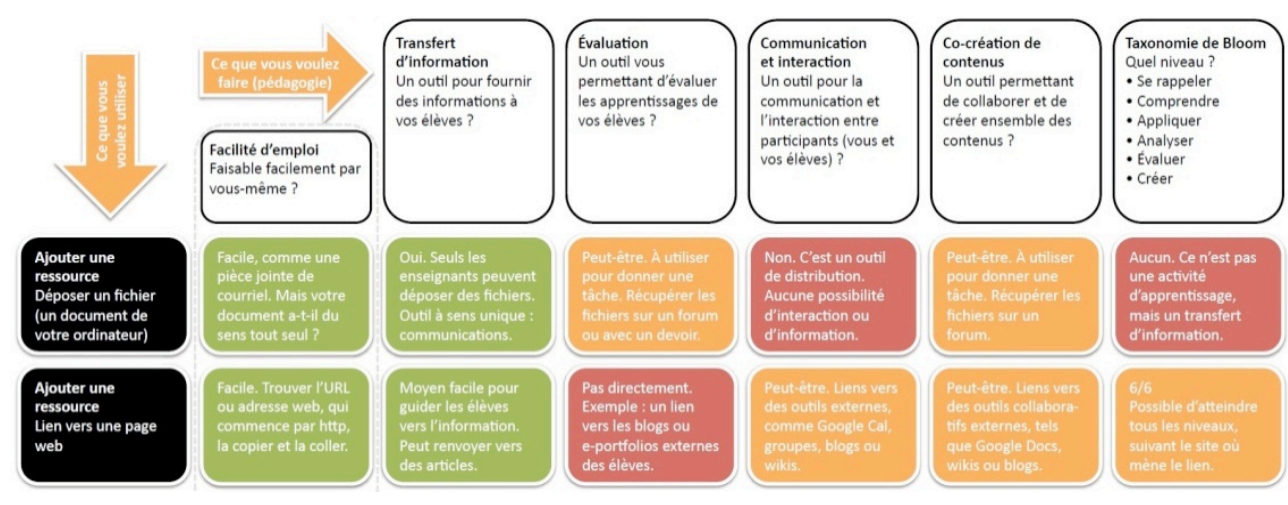

Publié sous licence Creative Commons

En premier lieu, on remarque la différence entre "ajouter une ressource» de type document numérisé (présente dans ses propres ressources) et utiliser un «lien ressource URL » qui peut renvoyer à des outils de collaboration (Google drive, par exemple). Alors que le premier cas confine à une transmission d'information, le second permet des interactions, la co-création de contenus et surtout l'atteinte des 6 niveaux de la taxonomie de Bloom et al.

En deuxième lieu, des nuances évoquées dans la figure 7 sont repérables. Il n’y a que très peu de différences entre «Forum de discussion» et «Wiki»; cependant, le caractère "peu adapté » selon la colonne «communication-interaction» du wiki devrait plutôt le destiner prioritairement à la co-création de contenus. L'astérisque ajouté à «Forum de discussion» au bas du tableau original renvoie à la nécessité d'être créatif. Dans l'hypothèse contraire, un forum peut rester effectivement un lieu d'échanges, relativement pauvre pédagogiquement. Martignoni propose des exemples : débats, discussions en équipe, rapports hebdomadaires, commentaires de ressources, jeux de rôle, etc. On ne peut qu'y voir l'exigence d'interactions « riches »

\footnotetext{
${ }^{20}$ Taxonomie des apprentissages cognitifs de Bloom et al. 1956.

$<\mathrm{http}$ ://www.cefes.umontreal.ca/ressources/guides/plan_cours/doc/taxonomie-cognitif.pdf $>$ (consulté le 15 novembre 2014).
} 
pour atteindre au moins les 5 niveaux sur 6 listés par Bloom et al. (comprendre, appliquer, analyser, évaluer, créer).

Figure 7. Tableau croisé Seitzinger-Martignoni (2)
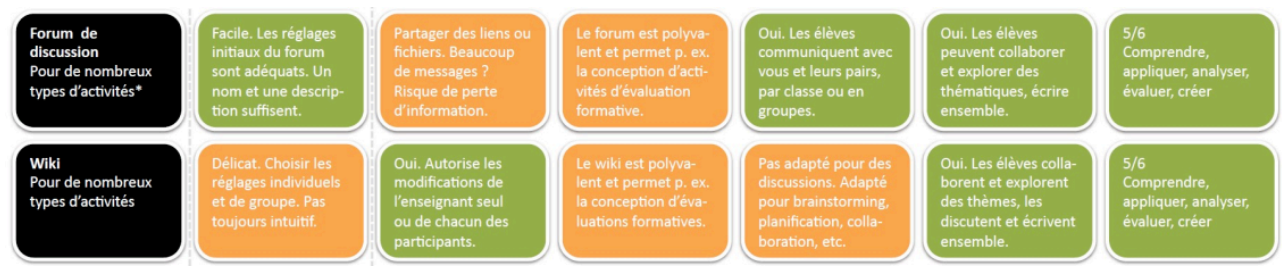

Publié sous licence Creative Commons

Même en ne sélectionnant que 2 parties réduites du tableau, son pragmatisme met en évidence non plus "un outil logiciel dédié à telle ou telle activité », mais des types d'activités, favorisant tel ou tel travail. Évaluer leurs possibilités selon l'échelle de Bloom et al. permet de mieux concevoir (et scénariser) une articulation entre opérations de bas et haut niveau. En outre, cette approche pragmatique suggère un rééquilibrage au profit du pôle " apprentissage » lors d'interactions et de création de contenus en commun.

\section{Conclusion}

L'exploitation de Moodle en Master DiFLES montre l'intérêt de construire une «culture » des outils Tice nourrie de pratiques concrètes. L'observation de ses usages lors de tentatives d'exploitation révèle la nécessité d'en dépasser les utilisations banales. Enrichir les compétences d'un utilisateur «éclairé » par la promotion d'une analyse propre au concepteur technico-pédagogique s'est avéré fructueux. Il ressort notamment de cette expérimentation, l'importance d'une scénarisation raisonnée, la prégnance de l'approche actionnelle et l'intérêt d'une démarche de type «pédagogie du projet $»$.

\section{Références}

Baudrit, A. 2007. "Apprentissage collaboratif, apprentissage coopératif: d'un comparativisme conventionnel à un comparativisme critique ». Les Sciences de l'Éducation-Pour l'Ére nouvelle, vol. 40, $n^{\circ} 1$ : 115-136.

Béguin, P. \& P. Rabardel. 2000. «Concevoir pour les activités instrumentées ». RIARevue d'intelligence artificielle, 14 (1-2) : 35-54.

Bergey, J.-L., V.-T. Nguyen \& H. Portine. 2012. « De l'apprenant-communiquant à l'apprenant-analyste: Quand les TICe font place à l'ingénierie linguistique ». Le langage et l'homme. Revue de didactique du français, vol. 47, $n^{\circ} 1: 49-60$.

Bloom, B. S. et al (eds.). 1956. Taxonomy of Educational Objectives: The Classification of Educational Goals. Handbook I: Cognitive Domain. New York : David Mc Kay. 
Charlier, B., N. Deschryver, \& D. Peraya. 2006. «Apprendre en présence et à distance : une définition des dispositifs hybrides ». Distances et Savoirs, 4 (4) : 469-496.

Conole, G. \& P. Alevizou. 2010. «A literature review of the use of Web 2.0 tools in Higher Education ». Report commissioned for the Higher Education Academy. [En ligne].

URL: <http://www.jisctechdis.ac.uk/assets/EvidenceNet/Conole_Alevizou_2010.pdf> (consulté le 15 décembre 2014).

Coquilhat, J.-C. 2008. "Mise à distance d'un enseignement de l'anglais de l'informatique : expérimentations et analyses de quelques aspects méta-didactiques et cognitifs de l'acquisition en anglais de spécialité ». Thèse de Doctorat, Université Bordeaux 2.

Downes, S. 2012. Connectivism and Connective Knowledge: Essays on Meaning and Learning Networks.

[En ligne]. URL : <http://www.downes.ca/files/books/Connective_Knowledge19May2012.pdf $>$ (consulté le 15 novembre 2014).

Foucault, M. 1988. "Les techniques de soi». In Dits et écrits, vol. 4. Paris : Gallimard, 783-813.

Guichon, N. 2011. «Former les futurs enseignants de langue en ligne par le biais de la rétrospection ». ALSIC, vol. 14. [En ligne]. URL : <http://alsic.revues.org/1983> (consulté le 15 novembre 2014).

Hubbard, P. 2008. "CALL and the future of language teacher education ». Calico Journal, 25(2) : 175-188.

Lebrun, M. 2011. «Impacts des TIC sur la qualité des apprentissages des étudiants et le développement professionnel des enseignants : vers une approche systémique ». STICEF, vol. 18. [En ligne]. URL : <http://sticef.univ-lemans.fr/num/vol2011/03rlebrun-tice/sticef_2011_lebrun_03rp.pdf $>$ (consulté le 15 novembre 2014).

Lebrun, M. 2014.Blogde M@rcel. $<$ http://lebrunremy.be/WordPress/?p=629\#comments $>$ (consulté le 15 novembre 2014).

Lewis, R. 1998. «Apprendre conjointement : une analyse, quelques expériences et un cadre de travail ». In Rouet, J.-F. \& B. de la Passardière (dir.). Actes du $4^{e}$ colloque « Hypermédias et apprentissages ». Paris : INRP, 11-28.

Lindsay, P. H. \& D. A. Norman. 1980. Traitement de l'information et comportement humain une introduction à la psychologie. Montréal : Éditions Études vivantes.

Martignoni, N. 2013. Guide des outils Moodle pour enseignant-e-s. fri-tic, Fribourg. $<$ https://moodle.org/pluginfile.php/1348613/mod_resource/content $/ 2 /$ MoodleTool Guide_fr.pdf $>$ (consulté le 15 novembre 2014).

Ollivier, $\bar{C} .2009$. « Mettre en œuvre une approche interactionnelle sur le web 2.0 ». In Lions-Olivieri, M.-L. \& P. Liria (dir.). L'approche actionnelle dans l'enseignement des langues. Paris : Éditions Maison des langues, 263-285.

Ollivier, C. \& L. Puren. 2011. Le web 2.0 en classe de langue. Paris : Éditions Maison des langues. 
Puren, C. 2009. «La nouvelle perspective actionnelle et ses implications sur la conception des manuels de langue ». In Lions-Olivieri, M.-L. \& P. Liria (dir.). L'approche actionnelle dans l'enseignement des langues. Paris : Éditions Maison des langues, 119-137.

Sarré, C. 2011. « Valeur ajoutée des plates-formes de téléformation pour les langues ». Les Langues Modernes, 2011/1:14-21.

Seitzinger, J. 2010. Moodle Tool guide For Teachers. EIT, Napier. $<$ http://www.catspyjamas.net/wpcontent/uploads/2010/05/MoodleToolGuideforTeachers_May2010_JS.pdf $>$

Stanford, J. 2009. Moodle 1.9 for Second Language Teaching. Birmingham : Packt Publishing.

Stockwell, G. 2012. " Mobile-assisted language learning ». In Thomas, M., H. Reinders \& M. Warschauer (eds). Contemporary Computer-assisted Language Learning. Londres : Bloomsbury Academic, 201-216.

Vaufrey, C. 2011. Livre blanc " TICE et scénarisation pédagogique ». Thot cursus éditions $<\mathrm{http} / /$ www.leslivresblancs.fr/societe/enseignement-et-formation/strategies-etmethodes/livre-blanc/tice-et-scenarisation-pedagogique-1375.html> (consulté le 25 novembre 2014).

Vaufrey, C. 2013. «Quelles plateformes et quels modèles d'enseignement pour notre culture numérique ?» <http://cursus.edu/article/19570/quelles-plateformes-quelsmodeles-enseignement-pour/\#.VICZCzGG-So> (consulté le 25 novembre 2014).

Zourou, K. 2012. «De l'attrait des médias sociaux pour l'apprentissage des langues Regard sur l'état de l'art». ALSIC, vol. 15, $n^{\circ} 1$. [En ligne]. URL : $<\mathrm{http}$ ://alsic.revues.org/2436> (consulté le 15 novembre 2014).

Actuellement doctorant et enseignant-contractuel à l'Université Bordeaux-Montaigne, JeanLuc Bergey a développé ses premières recherches en sciences du langage, après une dizaine d'années d'expérience de l'enseignement du FLE dans des contextes variés et une formation universitaire en Sciences du Langage. Il s'est spécialisé, après l'obtention d'un second Master en didactique de l'enseignement du FLE, dans le domaine du e-learning et notamment l'installation, l'administration et l'exploitation pédagogique de plateformes d'apprentissage. Son domaine d'intervention concerne la formation à la didactique des futurs enseignants de langue en intégrant l'ingénierie de la pédagogie et de la linguistique appliquées aux outils actuels des TICE.

$<$ jeanluc.bergey@gmail.com> 